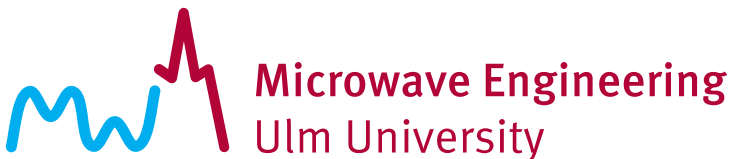

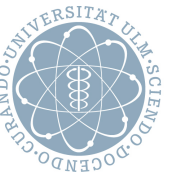

\section{Impact of an Automotive Chirp-Sequence Interferer on a Wideband OFDM Radar}

Christina Knill, Benedikt Schweizer, Philipp Hügler, and Christian Waldschmidt

(C) 2018 IEEE. Personal use of this material is permitted. Permission from IEEE must be obtained for all other uses, in any current or future media, including reprinting/republishing this material for advertising or promotional purposes, creating new collective works, for resale or redistribution to servers or lists, or reuse of any copyrighted component of this work in other works. 


\title{
Impact of an Automotive Chirp-Sequence Interferer on a Wideband OFDM Radar
}

\author{
Christina Knill $^{\# 1}$, Benedikt Schweizer ${ }^{\# 2}$, Philipp Hügler ${ }^{\# 3}$, and Christian Waldschmidt ${ }^{\# 4}$ \\ ${ }^{\#}$ Ulm University, Institute of Microwave Engineering, 89081 Ulm, Germany \\ $\left\{{ }^{1}\right.$ christina.knill, ${ }^{2}$ benedikt.schweizer, ${ }^{3}$ paul.huegler, ${ }^{4}$ christian.waldschmidt $\} @$ uni-ulm.de
}

\begin{abstract}
OFDM-based radar schemes are promising candidates to solve inherent limitations of frequency-modulated schemes. However, for a practical implementation in the automotive environment with an increasing amount of sensors and disturbances, not only the performance of the modulation scheme is important but also how it can perform in coexistence with other modulation schemes. In this paper, measurements are shown to investigate the impact of chirp-sequence-based sensors on a wideband OFDM radar. Furthermore, a straightforward method to mitigate and eliminate the interference on the receive signal is presented that allows reliable target detection even under severe chirp-sequence interference.
\end{abstract}

Keywords - automotive radar, interference, ofdm radar, radar measurements, signal processing

\section{INTRODUCTION}

In recent years, the number of automotive radars present in daily traffic has rapidly increased since the vehicles are increasingly equipped radar sensors. These sensors take on various tasks to support the driver or provide more comfort and safety on the road. Most of such radars use continuous waves or linearly frequency modulated waveforms with a trend towards shorter ramp durations (chirp-sequence). The influence of such sensors on each other is well investigated [1] and processing schemes to minimize the impact are known [2]-[4]. However, FMCW-based sensors have inherent limitations that might require different modulation approaches. Orthogonal frequency-division multiplexing (OFDM) is a prominent candidate for such digital modulation schemes. In contrast to a monofrequent signal, OFDM waveforms span the large measurement bandwidth at all time and have a relatively small power spectral density. Therefore, the influence of OFDM and FMCW based sensors on each other is different, but it must be known so that both approaches can operate side by side.

So far, the influence of OFDM on a chirp-sequence radar is barely known. In [5], the theoretical behavior is analyzed with simulations. In [6] and [7], mutual interference between two OFDM-based sensors is evaluated and correction methods based on thresholding and compressed sensing are presented.

The impact of a classical FMCW waveform on OFDM is determined in [8]. However, the measurements presented are coherently coupled and the narrowband interference assumption does not hold in case of short ramps with steep slopes. So far, the impact of chirp-sequence on OFDM radar is an open question that will be examined in this paper. Practical results of lab measurements are presented, analyzed, and a straightforward processing method that mitigates the impact of the interference is proposed and applied. The focus is on the analysis of measurement results, there will be neither simulations nor any theoretical interference signal models treated in the following.

\section{OFDM RADAR}

An OFDM radar frame is composed of $M$ OFDM symbols of duration $T$. The symbol duration in radar applications is typically in the range of a few microseconds. Each OFDM symbol comprises $N$ equidistant subcarriers $f_{n}$ with spacing $\Delta f=\frac{1}{T}$. To combat intersymbol interference due to channel path delays, a cyclic prefix is added to every OFDM symbol before transmission. For simpler depiction, the cyclic prefix is neglected in the following. The transmit signal yields in baseband domain

$$
x(t)=\sum_{m=0}^{M-1} \sum_{n=0}^{N-1} d_{m n} \mathrm{e}^{\mathrm{j} 2 \pi n \Delta f t} \operatorname{rect}\left(\frac{t-m T}{T}\right)
$$

where $d_{m n}$ is a symbol that is randomly selected from a modulation alphabet, e.g., QPSK, and transmitted on the $n^{\text {th }}$ subcarrier of the $m^{\text {th }}$ OFDM symbol. In case of reflection at a single target at distance $r_{0}$ with relative velocity $v$, the received OFDM frame in baseband domain (after removal of the cyclic prefix) yields

$$
\begin{aligned}
y(t)= & \sum_{m=0}^{M-1} \sum_{n=0}^{N-1} d_{m n} \mathrm{e}^{\mathrm{j} 2 \pi n \Delta f t} \\
& \times \mathrm{e}^{-\mathrm{j} 2 \pi\left(\frac{2 r_{0}}{c_{0}}\left(f_{c}+n \Delta f\right)\right)} \mathrm{e}^{\mathrm{j} 2 \pi\left(\frac{2 v m T}{c_{0}}\left(f_{c}+n \Delta f\right)\right)}
\end{aligned}
$$

with carrier frequency $f_{c}$. Attenuations due to free space or multi-path losses as well as the reflection are neglected.

Next, the signal is sampled using the Nyquist frequency $2 N \Delta f$. By element-wise division of the modulation terms, the received information of the $m^{\text {th }}$ symbol on the $n^{\text {th }}$ subcarrier is

$$
D(m, n)=\mathrm{e}^{-\mathrm{j} 2 \pi\left(\frac{2 r_{0}}{c_{0}}\left(f_{c}+n \Delta f\right)\right)} \mathrm{e}^{\mathrm{j} 2 \pi\left(\frac{2 v m T}{c_{0}}\left(f_{c}+n \Delta f\right)\right)} .
$$

The remaining phase terms are dominated by the target or channel information that can further be evaluated using 2D-Fourier transform resulting in the range-velocity or radar image. To regain reliable, distinct and undistorted target information, it is therefore required that preferable no phase terms or at least its majority are not violated or altered by interferences. 
Table 1. Chirp-sequence interferer signal properties

\begin{tabular}{c|c|c}
\hline Bandwidth $(\mathrm{GHz})$ & $B_{\text {chirp }}$ & 2 \\
Up-ramp time $(\mu \mathrm{s})$ & $T_{\text {chirp }}$ & 50 \\
Chirp repetition time $(\mu \mathrm{s})$ & $T_{\text {chirp,c }}$ & 60 \\
Number of ramps & $N_{\text {chirp }}$ & 258 \\
\hline
\end{tabular}

Table 2. OFDM radar signal properties

\begin{tabular}{c|c|c}
\hline Number of symbols & $M$ & 2048 \\
Number of subcarriers & $N$ & 2048 \\
Bandwidth $(\mathrm{GHz})$ & $B$ & 1.024 \\
Subcarrier spacing $(\mathrm{kHz})$ & $\Delta f$ & 500 \\
Symbol time $(\mu \mathrm{s})$ & $T$ & 2 \\
Cyclic prefix time $(\mu \mathrm{s})$ & $T_{c p}$ & 0.4 \\
Maximum range $(\mathrm{m})$ & $r_{\max }$ & 60 \\
Range resolution $(\mathrm{m})$ & $\Delta r$ & 0.15 \\
Maximum velocity $(\mathrm{m} / \mathrm{s})$ & $v_{\max }$ & \pm 50 \\
Velocity resolution $(\mathrm{m} / \mathrm{s})$ & $\Delta v$ & 0.39 \\
\hline
\end{tabular}

\section{CHIRP-SEQUENCE INTERFERENCE}

The interfering radar is a state-of-the-art chirp-sequence radar with chirp repetition time $T_{\text {chirp,c }}=60 \mu$ s and bandwidth $B_{\text {chirp }}=2 \mathrm{GHz}$. More signal details are listed in Table 1 . The center frequency is $76.5 \mathrm{GHz}$ and the equivalent isotropically radiated power (EIRP) is $17 \mathrm{dBm}$.

For the OFDM radar, the lab demonstrator presented in [9] is used. It has an EIRP of $8.5 \mathrm{dBm}$. Detailed properties of the transmitted OFDM signal are summarized in Table 2. To validate different amounts of the interference impact, the carrier frequency of the OFDM signal is set to $f_{c}^{(I)}=77 \mathrm{GHz}$ or $f_{c}^{(I I)}=76 \mathrm{GHz}$ resulting in an interfered bandwidth of $B / 2$ and $B$, respectively, as sketched in Fig. 1. Three different kinds of measurements depending on the amount of interference are distinguished in the following: none (none-interfered OFDM), half $\left(\mathrm{OFDM}^{(I)}\right.$ at $\left.77 \mathrm{GHz}\right)$, and full $\left(\mathrm{OFDM}^{(I I)}\right.$ at $\left.76 \mathrm{GHz}\right)$.

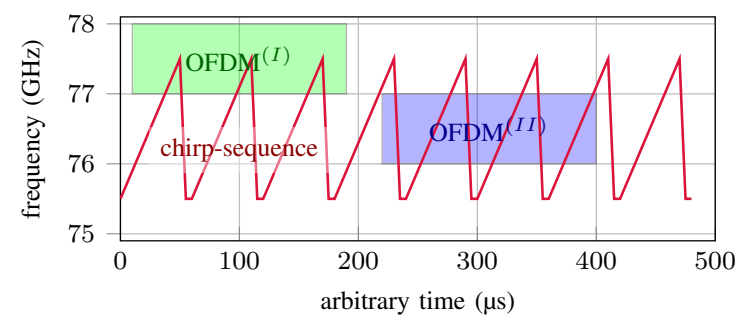

Fig. 1. Schematic diagram of the investigated and measured signal constellations for OFDM and chirp-sequence.

During measurements, both radars are not coherently connected or mutually triggered but run independently. In the measurement setup, both sensors face each other where the interferer is also equipped with a corner reflector representing a target to the OFDM radar, as shown in Fig. 2.

Measurements at $76 \mathrm{GHz}$ and $77 \mathrm{GHz}$ with and without chirp-sequence interference are performed in an anechoic

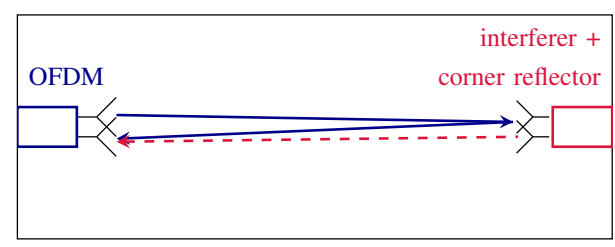

Fig. 2. Sketch of the measurement scene comprising the two radars.

chamber and then analyzed in the time-frequency and range-velocity domain. In Fig. 3(a) to (c), the time-frequency domain signal $D(m, n)$ (3) of the first 256 OFDM-symbols are shown for the three cases none, half, and full interference. Fig. 3a (none) shows the desired time-frequency signal figure. In Fig. 3b (half) and 3c (full), the fast ramps of the interfering signal are distinctly discernible as slightly slanted vertical lines of much higher amplitude. Considering the signal properties in Table 1 and 2, the expected number of interfered adjacent subcarriers per OFDM symbol is

$$
\frac{B_{\text {chirp }}}{T_{\text {chirp,up }}} \frac{T}{\Delta f}=160
$$

which is about $0.078 N$. Since multiple adjacent subcarriers instead of multiple adjacent symbols per subcarrier are affected, the interference cannot be treated as narrowband.

The range-velocity images of the signals in Fig. 3 are shown in Fig. 4. In the none-interfered case in Fig. 4(a), the target at $r=8.4 \mathrm{~m}$ and $v=0 \mathrm{~m} / \mathrm{s}$ is distinguishable from noise. With increasing interference impact (cf. 4(b) and 4(c)), the SNR distinctly decreases making target detection considerably more difficult. This circumstance is also observable in Fig. 5, that shows a comparison of the range-profiles of the target. With increasing interference, the noise level increases significantly.

The drop of SNR is further observable in the amplitude density distribution of the linear range-velocity signal which is presented in Fig. 6. The frequencies of occurrence of the amplitudes follow a Rayleigh distribution where the better the $\mathrm{SNR}$, the narrower is its shape, as it is for the non-interference case. The distributions of the interfered signals exhibit considerably flatter and wider shapes which indicates a significant decrease of the SNR. Without countermeasures to these effects, severe interference impact may have detrimental consequences for target estimation. Weaker targets will sink below the noise level and cannot be detected any more.

\section{INTERFERENCE Mitigation}

The increase of the noise level with increasing amount of interference is largely dominated by the significantly higher input power level of the interference signal per affected OFDM element $D(m, n)$ compared to its undisturbed signal level. This is due to the interference signal traveled only half the signal path compared to the reflected OFDM signal and therefore experienced less attenuation. Furthermore, the output power of the chirp-sequence interferer is concentrated in a much smaller instantaneous bandwidth where the OFDM output power is 


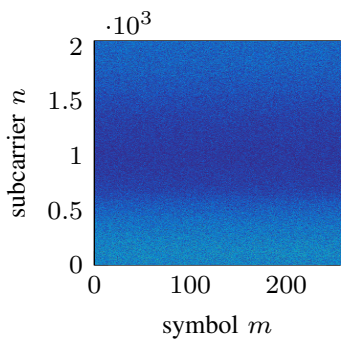

(a)

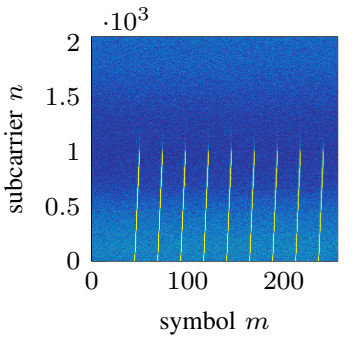

(b)

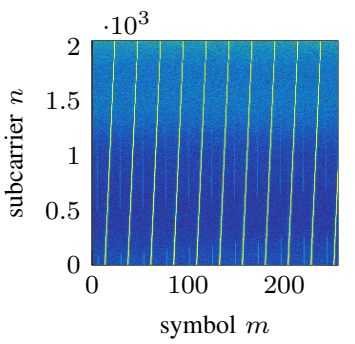

(c)

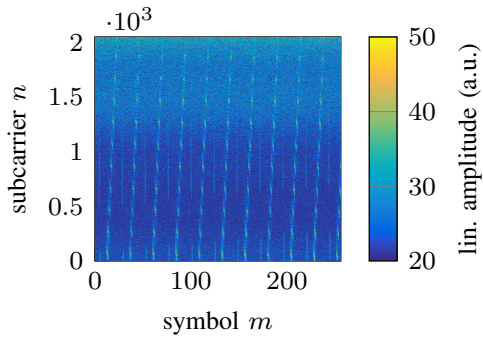

(d)

Fig. 3. Time-frequency domain signal (3) of the first 256 OFDM symbols for (a) non-interfered, (b) half- and, (c) full-bandwidth interfered OFDM, as well as

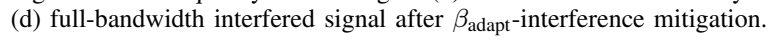

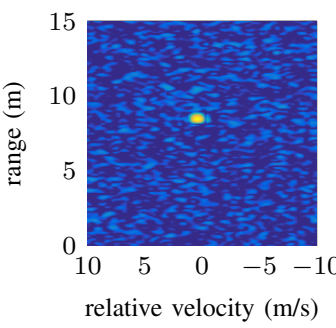

(a)

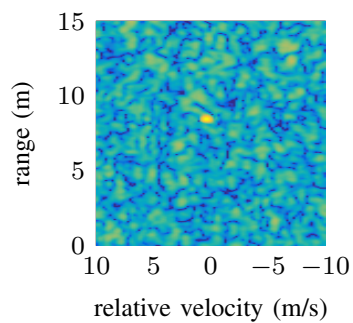

(b)

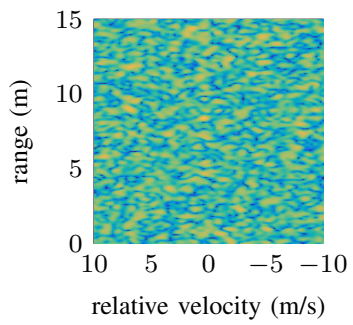

(c)

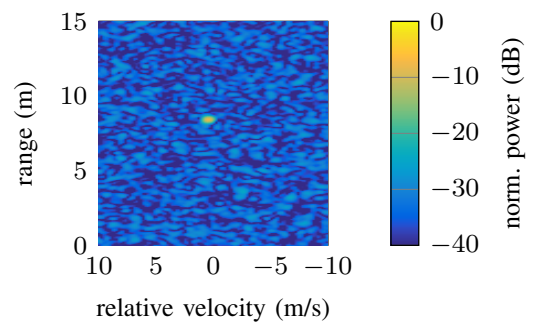

(d)

Fig. 4. Range-velocity images for (a) non-interfered, (b) half- and, (c) full-bandwidth interfered OFDM, as well as (d) full-bandwidth interfered signal after $\beta_{\text {adapt }}$-interference mitigation.

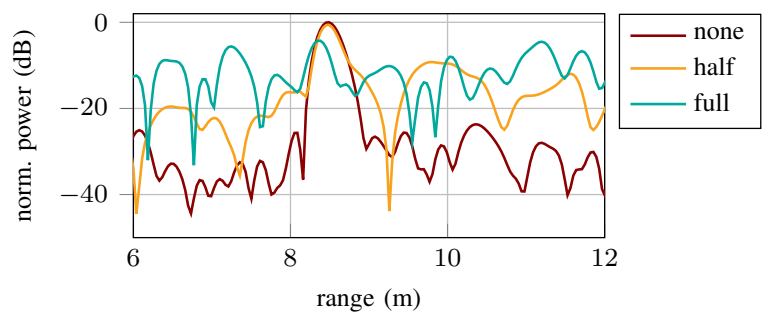

Fig. 5. Range-profiles at $v=0 \mathrm{~m} / \mathrm{s}$ for different interfered signals.

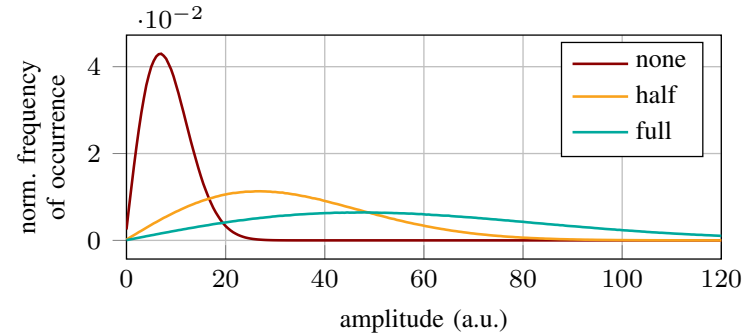

Fig. 6. Normalized frequency of occurrence of amplitudes (linear) in range-velocity domain.

spread over multiple subcarriers (small spectral power density) which leads to a smaller signal level per element. To combat these impacts, an interference mitigation post-processing is performed. Since it is impossible to eliminated the interference in analogue domain before $\mathrm{AD}$-conversion, this is done in digital. The chirp-sequence interference is identifiable by its

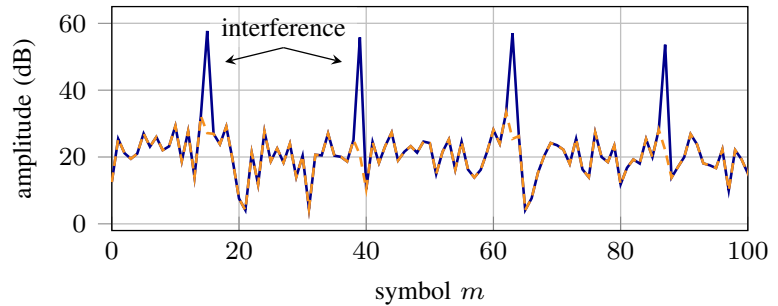

Fig. 7. Extract of the interfered (- $\longrightarrow$ ) and adapted (----) signal amplitude of a single subcarrier along multiple symbols.

sharp ridges along multiple carriers (cf. Fig. 7), but only covering very few adjacent OFDM symbols. Compared to the adjacent local average signal level, an interfered element shows a significant deviation of its amplitude which is exploited for fast interference detection. A straightforward approximation of the local signal average is accomplished by convolving the time-frequency signal (3) with a local filter mask, e.g.,

$$
F=\left[\begin{array}{lllllll}
1 & 1 & 1 & 0 & 1 & 1 & 1
\end{array}\right] / 6,
$$

which determines the average signal level $\bar{D}(m, n)$ along consecutive elements of the same subcarrier using three previous and three subsequent elements. It should be noted that this specific filter mask is just an example and may not be suitable for all cases of interference, e.g. several interferers.

A threshold $\alpha|\bar{D}(m, n)|$ proportional to $\bar{D}(m, n)$ and a constant $\alpha \geq 1$ is applied to identify interfered elements. Then, 
interference mitigation $\beta(m, n)$ is applied to them which yields the interference mitigated signal

$$
\hat{D}(m, n)=\left\{\begin{array}{ll}
\beta(m, n), & \text { if }|D(m, n)| \geq \alpha|\bar{D}(m, n)| \\
D(m, n), & \text { otherwise }
\end{array} .\right.
$$

The simplest realization of mitigation is replacing interfered elements by zeros (canceling). However, deleting elements not only eliminates the interference but also the OFDM signal phase information and reduces the processing gain. Another possibility is adapting the signal amplitude using

$$
\beta_{\text {adapt }}(m, n)=D(m, n) \frac{|\bar{D}(m, n)|}{|D(m, n)|},
$$

where the amplitude is adjusted to $|\bar{D}(m, n)|$. This method preserves the signal phase and only adjusts the amplitude of the element.

In Fig. 7, the signal of one subcarrier with applied interference mitigation using (7) is compared to its interfered signal. Besides the mitigated interferences, the signals are similar. Time-frequency and range-velocity profiles for the full interference case with applied signal level adjustment are presented in Fig. 3(d) and Fig. 4(d), respectively. In both domains, the impact of the interference is reduced and target detection is facilitated. Fig. 8 and 9 validates that the SNR for both half and full interference is increased approaching the non-interfered SNR.

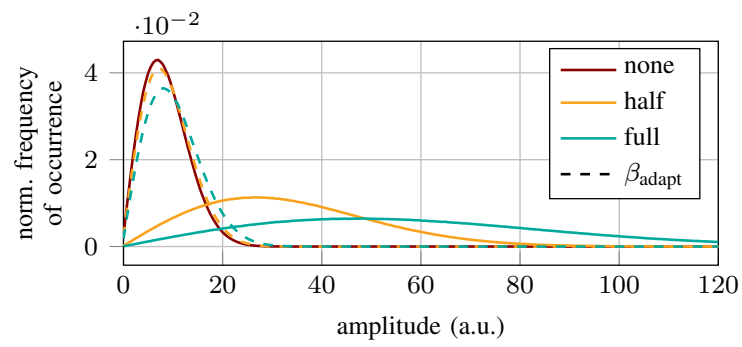

Fig. 8. Normalized frequency of occurrence of amplitudes (linear) in range-velocity domain before and after interference mitigation.

\section{CONClusion}

In this paper, measurements investigating the impact of an automotive chirp-sequence radar on a wideband OFDM radar are presented and evaluated for the first time. Chirp-Sequence interferences cause sharp ridges along multiple carriers but only covering very few adjacent OFDM symbols in the time-frequency-signal. This leads to a decrease of SNR proportional to the amount of interference. To avoid the loss of SNR, straightforward interference mitigation method is proposed which restore the OFDM signal level and SNR such that it is similar to a non-interfered OFDM signal.

\section{ACKNOWLEDGMENT}

The research leading to these results was conducted within the Tech Center a-drive and was funded by the MWK Baden-Württemberg. Responsibility for the information and views set out in this publication lies entirely with the authors.

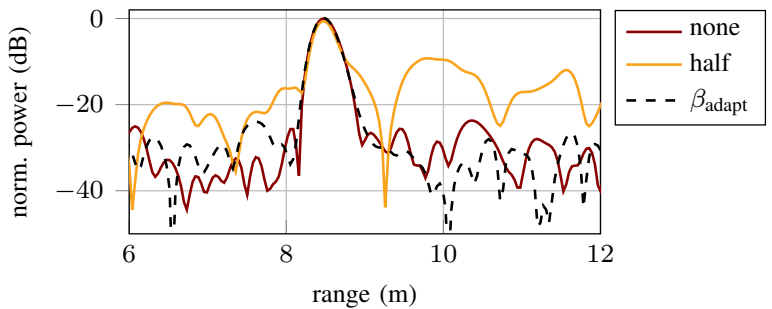

(a)

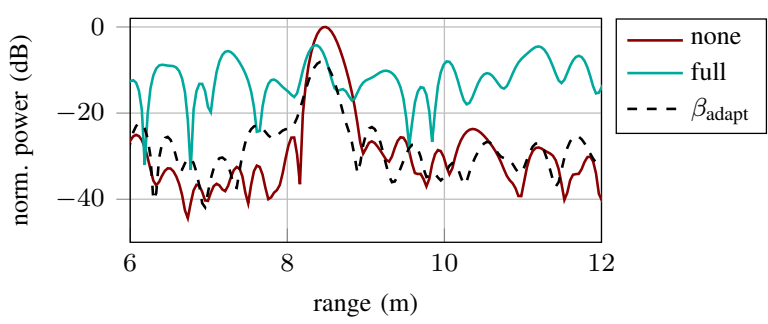

(b)

Fig. 9. Range-profiles at $v=0 \mathrm{~m} / \mathrm{s}$ before and after interference mitigation for (a) half and (b) full bandwidth interfered OFDM.

\section{REFERENCES}

[1] T. Schipper, M. Harter, T. Mahler, O. Kern, and T. Zwick, "Discussion of the operating range of frequency modulated radars in the presence of interference," International Journal of Microwave and Wireless Technologies, vol. 6, pp. 371-378, 2014.

[2] M. Barjenbruch, D. Kellner, K. Dietmayer, J. Klappstein, and J. Dickmann, "A Method for Interference Cancellation in Automotive Radar," in IEEE MTT-S International Conference on Microwaves for Intelligent Mobility (ICMIM), Apr. 2015.

[3] J. Bechter, K. Eid, F. Roos, and C. Waldschmidt, "Digital Beamforming to Mitigate Automotive Radar Interference," in IEEE MTT-S International Conference on Microwaves for Intelligent Mobility (ICMIM), May 2016.

[4] J. Bechter, C. Sippel, and C. Waldschmidt, "Bats-Inspired Frequency Hopping for Mitigation of Interference Between Automotive Radars," in 2016 IEEE MTT-S International Conference on Microwaves for Intelligent Mobility (ICMIM), May 2016.

[5] C. Knill, J. Bechter, and C. Waldschmidt, "Interference of Chirp Sequence Radars by OFDM Radars at $77 \mathrm{GHz}$," in IEEE MTT-S International Conference on Microwaves for Intelligent Mobility (ICMIM). IEEE, Mar. 2017, pp. 147-150.

[6] Y. L. Sit and T. Zwick, "MIMO OFDM Radar Networks: Inter\& Intra-System Interference Handling," in Asia-Pacific Microwave Conference, Nov. 2014, pp. 1318-1320.

[7] B. Nuss and T. Zwick, "A Novel Interference Mitigation Technique for MIMO OFDM Radar Using Compressed Sensing," in European Radar Conference (EURAD), 2017, pp. 98-101.

[8] G. Hakobyan and B. Yang, "A Novel Narrowband Interference Suppression Method for OFDM Radar," in 24th European Signal Processing Conference (EUSIPCO). IEEE, Aug. 2016, pp. 2230-2234.

[9] B. Schweizer, C. Knill, D. Schindler, and C. Waldschmidt, "Stepped-Carrier OFDM-Radar Processing Scheme to Retrieve High-Resolution Range-Velocity Profile at Low Sampling Rate," IEEE Transactions on Microwave Theory and Techniques, vol. 66, no. 3, pp. 1610-1618, Mar. 2018. 